\title{
Antihelmintic Treatment And Strategy To Eliminate Soil-Transmitted Helminth In Children
}

\author{
Sacharissa Zerlina Tsarwah Thirafi ${ }^{1(\mathbb{D})}$, Zaky Firmawan El-Hakim ${ }^{1}{ }^{\mathbb{D}}$, Lutfi Dewanda \\ Nugroho $^{1}{ }^{\mathbb{D}}$, Ayush Khangai ${ }^{2,3}$, Dwiyanti Puspitasari ${ }^{* * \mathbb{D}}$
}

${ }^{1}$ Faculty of Medicine, Universitas Airlangga, Surabaya, Indonesia

${ }^{2}$ Department of Environmental and Preventive Medicine, Oita Univerisity, Japan

${ }^{3}$ The First Central Hospital of Mongolia

${ }^{4}$ Department of Pediatrics, Dr. Soetomo General Academic Hospital - Faculty of Medicine, Universitas Airlangga,

Surabaya, Indonesia

\section{A R T I C L E I N F O}

\section{Article history:}

Received 03 March 2021

Received in revised form 28 May 2020

Accepted 25 June 2021

Available online 30 June 2021

Keywords:

Soil-Transmitted Helminth,

Elimination,

Transmission,

Children.

*) Corresponding author: dwiyanti-p@fk.unair.ac.id

\begin{abstract}
A B S T R A C T
Introduction: Soil-transmitted helminthiasis (STH) is one of the neglected tropical diseases. These worm infections contributed to global child health. Children suffer from chronic disease and fail to reach their full physical and intellectual capacity. Antihelmintic treatment or deworming is recommended for controlling STH. World Health Organization is focusing predominantly on deworming school-age children. Even antihelmintic treatment has to be repeated regularly, children can reinfect if their environment contaminated. It needs a strategy to control STH and treating adults as well as a child.

Methods: We searched the literature for relevant clinical research and articles using Google Scholar and PubMed which were published between 2014 and 2018 with keywords "soil-transmitted helminths", "soil-transmitted helminthiasis", combined with "eradication" and "elimination". We excluded research and article which were not written in English. Our study included a total number of ten publications.

Results: The studies mentioned that antihelmintic requires the control of STH transmission and need for the treatment in different age groups.

Conclusion: According to our findings, the mass drug administration is recommended as a control strategy in both low and middle-income countries to reduce helminthiasis and schistosomiasis. High STH reinfection rate requires an integrated approach including the use of safe pure water, improvement for personal hygiene and sanitation, proper toilets, and reducing the practice of defecation in nature. The previous studies showed that studies focused on deworming using antihelmintic
\end{abstract}

\section{Introduction}

Soil-transmitted helminthiasis (STH) is an infection of parasitic worms, such as roundworms (Ascaris lumbricoides), whipworms (Trichuris trichiura), or hookworms (Necator americanus and Ancylostoma duodenale). A. duodenale is less common than $N$. americanus. A. duodenale generally present in concomitant with $N$. americanus. ${ }^{1}$ Based on WHO classification, STH was classified into Neglected Tropical Diseases (NTDs). Globally, there are 1.4 billion people affected.2 Helminth infections caused by STH and schistosomes are one of the most prevalent NTDs in poverty settings. ${ }^{3}$ Most of the cases were located in Southeast Asia. ${ }^{2}$ STH was considered a neglected tropical disease because of the three main features:

1. More prevalent in underdeveloped countries.

2. Chronic disease, not an acute disease.

3. The effect of this infection on economic and education burden is not quantified. ${ }^{4}$

Due to this infection, the burden mostly fell on school-age children (SAC) in developing countries (although in hookworm infections, the infection rate increases with age and plateau in adulthood). ${ }^{3}$ According to the report of the World Bank's morbidity in children,

Biomolecular and Health Science Journal

Available at https://e-journal.unair.ac.id/BHSJ DOI: 10.20473/bhsj.v4i1.25947 
there is helminth infection between $20 \%$ of cases under 14 years old who are in unable to self-caring life years. Previous studies suggested an association between helminth infection and their academic achievement and cognitive. ${ }^{5}$ In developing countries, helminthiasis and schistosomiasis are the most prevalent pediatric infections. These chronic infections have significant consequences in both growth and development. ${ }^{6}$ Other high-risk groups susceptible to morbidity caused by STH were preschool-aged children (PSAC) and women of reproductive age (WRA), but these groups did not get attention as much as SAC. . $^{5,7}$

Soil-transmitted helminth infection was a longstanding public health problem, particularly in developing countries. The higher prevalence found in rural low socioeconomic populations. ${ }^{6}$ Other attributes of helminth infection were rapid reinfection after treatment without significant acquired immunity and an aggregated distribution (few individuals harbor a high number of parasites). ${ }^{3}$ Although a single or mixed soil-transmitted helminth infection rarely caused death, nutritional status, growth, cognitive development, and human health were greatly impacted, particularly in children. ${ }^{6}$

WHO recommended an integrated approach to the control of neglected tropical diseases to avoid duplication of effort and to reduce costs. ${ }^{8}$ WHO recommended a control strategy through mass drug administration (MDA). MDA, education, and other non-medication health measures were perceived to be more important for STH elimination than vector control and the development of a new tool. ${ }^{9}$ The goal was, by 2020, endemic countries increase their antihelmintic coverage to $75 \%$ in both pre-school- and school-age children. ${ }^{7}$ The remaining question was the possibility of disease-eradication in certain circumstances. Another suggestion for the elimination of STH offered a chance to eliminate STH-related morbidity significantly while decreasing the need for resources for NTD. ${ }^{10,11}$ This literature review aimed to show several studies that help to reduce the morbidity of STH.

\section{Discussion}

\section{Antihelmintic Treatment}

According to the United States and other non-endemic countries recommendations such as Europe and Japan on pediatric helminth infection, children affected by the disease need to be treated after diagnosed specifically. ${ }^{4}$ Albendazole, the first-line single-dose drug does not absorb outside the intestine and is only used for ascariasis and enterobiasis cases. ${ }^{4}$ Longer course albendazole or mebendazole are used for trichuriasis while mebendazole could also be used in hookworm treatment. Albendazole combined with ivermectin can also treat trichuriasis. ${ }^{4}$ The major limitation of this program was the emergence of drug resistance in parasites. $^{9}$

Through MDA programs, children in developing countries Asia, Africa, and America recommended to receive therapy for helminthiasis, schistosomiasis, or mixed infection. Early in 2001, MDA recommended developing countries and nongovernmental agencies to give a single dose of either albendazole (400 $\mathrm{mg})$ or mebendazole $(500 \mathrm{mg})$ combined with praziquantel annually (administered as multiple tablets depending on the use of a height pole)., ${ }^{42}$

Mass deworming (community-wide) significantly reduces the STH prevalence for both hookworm and ascariasis in school-age children than targeted deworming (children only). ${ }^{13}$ This suggests that improving morbidity outcomes were likely to be achieved by deworming programs at the community level to minimize the incidence in the high-risk population, such as school-age children.

WHO informed only $30 \%$ of children who need albendazole or mebendazole treatment are receiving antihelminth drugs. The deworming was further supported by London Declaration for Neglected Tropical Disease 2012. Major pharmaceutical companies reconfirmed their commitment in this declaration to donate antihelmintic drugs. Due to continuing transmission and high reinfection rates, currently, there is no consensus on whether this strategy achieved the eradication/elimination of helminthiasis or schistosomiasis. Programs for hygiene, sanitation, and pure water were critical to achieving control. ${ }^{4,12-14}$ Education programs had an important role as the strategy mentioned above needs community-level awareness to enhance the efficiency of the helminth elimination program. It was important to employ strategies that debunk misconceptions and myths about the disease. ${ }^{15}$

\section{Integrated Approach}

An integrated approach plays an important role in longterm success, such as a large-scale community-based program, considering that deworming only did not resolve the problem. ${ }^{16-18}$ Mass deworming in children significantly reduces the overall transmission in the community, however, the high rate of reinfection is decreasing as the advantages obtained by deworming. ${ }^{16,19}$ Research showed the prevalence of $A$. lumbricoides, $T$. trichiura, and hookworms returned to $26 \%, 36 \%$, and $30 \%$ after three months, and one year after the treatment, the prevalence rate was restored to $94 \%, 82 \%$, and $57 \%$, respectively. ${ }^{20}$

The possibility of going from regulation to an elimination strategy existed. Controlling STH decreased the severity of infections in targeted populations, resulting in lower morbidity. ${ }^{10}$ Adults were rarely targeted, but they were critical transmitters for reinfection in children who have been treated. According to recent models, transmission disruption could be possible by extended community-wide MDA process, which has been demonstrated to be feasible by other programs. ${ }^{10}$ High standards of coverage and enforcement are needed. The risk of stopping premature STH programs that might lead to a danger of antihelminthic resistance are potential challenges in the future. ${ }^{10}$

Human mobility is also a factor affecting STH spreading, thus it also affecting STH elimination programs. It is recommended that individuals arriving from areas with high STH transmission should receive chemotherapy for STHs. This could be most easily implemented if the migration is seasonal and coincides with treatment rounds. Transmission hotspots in treatment groups should be eliminated, for example, by implementing appropriate water, sanitation, and hygiene measures (WASH) and targeted treatment of 
individuals living in hotspots. ${ }^{21}$

To enhance the STH elimination program, strong leadership, international cooperation, and the allocation of appropriate resources, infrastructure and staff qualified for data collection, analysis and reporting are required. Unfortunately, in developing countries, where many of these diseases are widespread, these are often poor or non-existent because of a lack of funds. There is an urgent need to coordinate/control activities to reliably diagnose global disease conditions and compare results from different countries. A major step forward in this regard was the establishment of an international database, regularly updated, providing open access to all verified research data. ${ }^{22}$ It is also noted that diagnostic tools must be developed in response to epidemiological changes of STH transmission levels in the era of preventive chemotherapy, thus urging researchers to develop a new STH diagnostic tool. ${ }^{23}$

\section{Treatment Guideline and Strategy}

The current recommendation regarding a $50 \%$ increase of any STH prevalence in school-age children needs several updates. ${ }^{16,19,24,25}$ Deworming program almost exclusively targets school children because of cost issues and school children are the group with a high risk of morbidity, ${ }^{7,24,26}$ but treating all age-group was more recommended than treating children twice a year in areas where hookworm infection was predominant since adults still harbor parasites thus maintaining transmissions. ${ }^{24,27}$ It was efficient in limited-resource settings for the long term. The best choice in areas with high-transmission Ascaris was to increase frequency while also broadening coverage across all age groups. ${ }^{16,19,24}$

Figure 1 compared mass drug administration methods focused on once or twice-yearly treatment for both pre-school- and school-age children. ${ }^{24}$ In terms of decreasing the overall burden of Ascariasis, a higher frequency of mass drug administration in children was expected to be marginally more effective than annual mass treatment. However, this method has little effect on the hookworm. ${ }^{24}$ Treating only children did not affect the level of transmission. ${ }^{24}$

To assess the effectiveness of drug distribution in the MDA program, the standard is an extensive parasite stool examination. A study in the Zanzibar region suggests that health record analysis could help in assessing parasite distribution. This approach would not require "gold standard" parasitological stool examination, which is costly and not applicable on a large scale. This method's sensitivity will be compromised by MDA. ${ }^{28}$

\section{Ascaris}

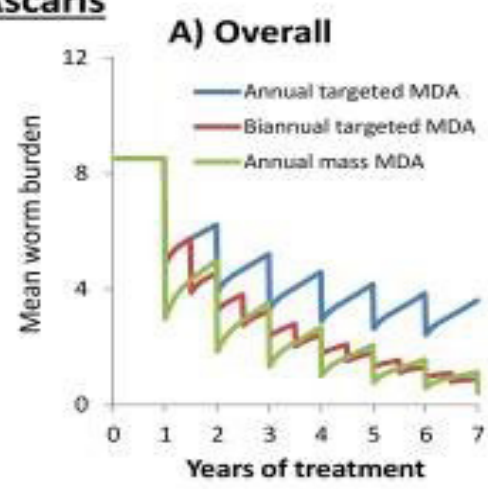

\section{Hookworm}

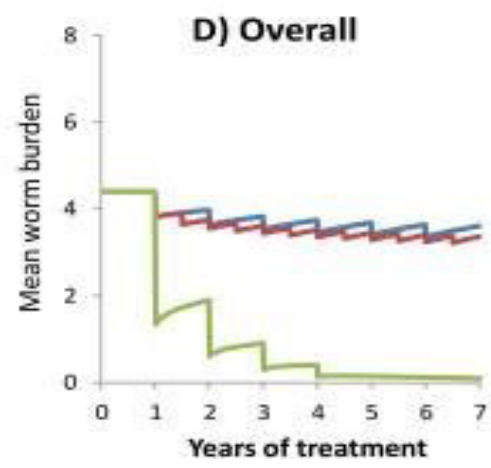

B) Children
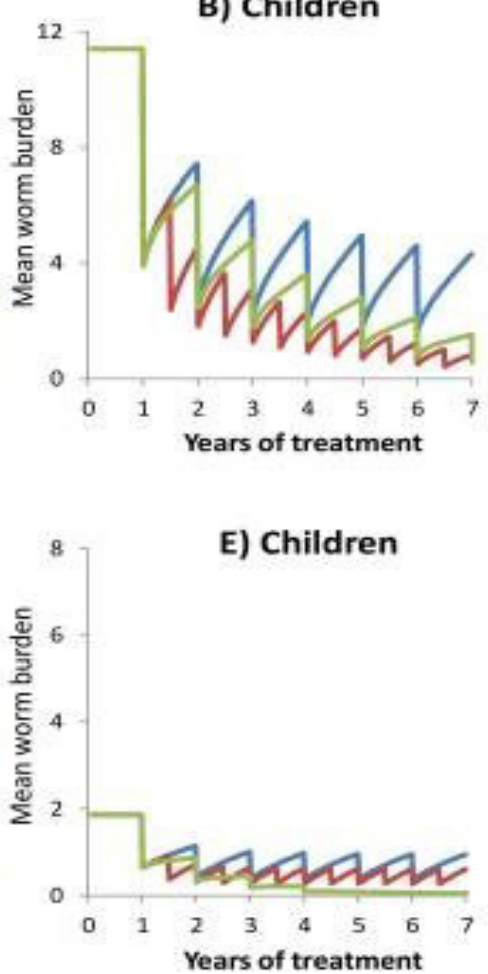

C) Adults
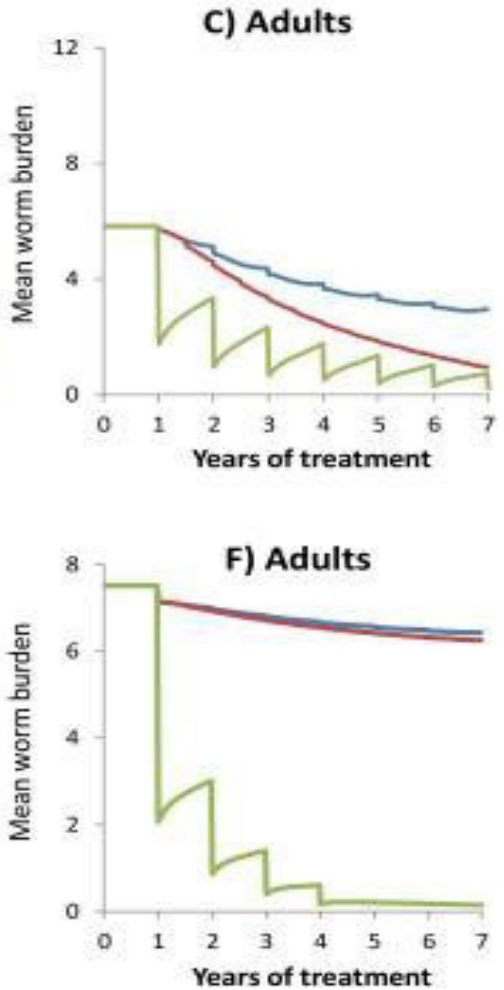

Figure 1. Impact of different treatment on the mean number of worms in different age grouping24 


\section{Conclusion}

Mass drug administration is recommended as a control strategy in both low and middle-income countries to reduce helminthiasis and schistosomiasis. High STH reinfection rate requires an integrated approach including the usage of safe pure water, improvement of personal hygiene and sanitation, proper toilets, and reducing the practice of defecation in nature. The reference studies focused on deworming via antihelmintic to eliminate soil-transmitted helminthiasis. Instead of treating children only, these studies suggest a new strategy of broad treatment across all ages group. Also, previous studies are showing the result of stopping transmission in the soil-transmitted helminth. Eventually, we need to find the best treatment strategy to eliminate STH.

\section{Conflict of Interest}

The author stated there is no conflict of interest

\section{References}

1. Wibawa T, Satoto TBT. Magnitude of Neglected Tropical Diseases in Indonesia at Postmillennium Development Goals Era. J Trop Med; 2016. Epub ahead of print 2016. DOI: 10.1155/2016/5716785.

2. Dunn JC, Turner HC, Tun A, et al. Epidemiological surveys of, and research on, soil-transmitted helminths in Southeast Asia: A systematic review. Parasites and Vectors 2016; 9: 1-13.

3. Mekete K, Ower A, Dunn J, et al. The Geshiyaro Project: A study protocol for developing a scalable model of interventions for moving towards the interruption of the transmission of soil-transmitted helminths and schistosome infections in the Wolaita zone of Ethiopia. Parasites and Vectors 2019; 12: 1-12.

4. Weatherhead JE, Hotez PJ. Worm Infections in Children. Pediatr Rev 2015; 36: 341-354.

5. Freeman MC, Akogun O, Belizario V, et al. Challenges and opportunities for control and elimination of soil-transmitted helminth infection beyond 2020. PLoS Negl Trop Dis 2019; 13: 1-10.

6. Simarmata N, Sembiring T, Ali M. Nutritional status of soiltransmitted helminthiasis-infected and uninfected children. Paediatr Indones 2015; 55: 136-41.

7. Becker SL, Liwanag HJ, Snyder JS, et al. Toward the 2020 goal of soil-transmitted helminthiasis control and elimination. PLoS Negl Trop Dis 2018; 12: 1-17.

8. World Health Organization. Assessing the epidemiology of soiltransmitted helminths during a transmission assessment survey in the global programme for the elimination of lymphatic filariasis. World Health Organization, 2015.

9. Keenan JD, Hotez PJ, Amza A, et al. Elimination and Eradication of Neglected Tropical Diseases with Mass Drug Administrations: A Survey of Experts. PLoS Negl Trop Dis 2013; 7: 1-8.

10. Ásbjörnsdóttir KH, Means AR, Werkman M, et al. Prospects for elimination of soil-transmitted helminths. Curr Opin Infect Dis 2017; 30: $482-488$.

11. Truscott JE, Turner HC, Anderson RM. What impact will the achievement of the current World Health Organisation targets for anthelmintic treatment coverage in children have on the intensity of soil transmitted helminth infections? Parasites and Vectors 2015; 8: $1-12$.

12. Campbell SJ, Nery S V, McCarthy JS, et al. A Critical Appraisal of Control Strategies for Soil-Transmitted Helminths. Trends Parasitol 2016; 32: 97-107.
13. Clarke NE, Clements ACA, Doi SA, et al. Differential effect of mass deworming and targeted deworming for soil-transmitted helminth control in children: a systematic review and meta-analysis. Lancet 2017; 389: 287-297.

14. Anderson R, Truscott J, Hollingsworth TD. The coverage and frequency of mass drug administration required to eliminate persistent transmission of soil-transmitted helminths. Philos Trans R Soc B Biol Sci 2014; 369: 20130435-20130435.

15. Sacolo-Gwebu H, Kabuyaya M, Chimbari M. Knowledge, attitudes and practices on schistosomiasis and soil-transmitted helminths among caregivers in Ingwavuma area in uMkhanyakude district, South Africa. BMC Infect Dis 2019; 19: 1-11.

16. Devnani M. An integrated approach is the key to control of soiltransmitted helminths. Br Med J; 350, https:/www.bmj.com/ content/350/bmj.h720/rr (2015).

17. Mudur G. Deworming programme in India sparks controversy. BMJ Br Med J 2015; 350: h720

18. Taylor-Robinson DC, Maayan N, Soares-Weiser K, et al. Deworming drugs for soil-transmitted intestinal worms in children: Effects on nutritional indicators, haemoglobin, and school performance. Cochrane Database Syst Rev; 2015. Epub ahead of print 2015. DOI: 10.1002/14651858.CD000371.pub6.

19. Truscott JE, Hollingsworth TD, Brooker SJ, et al. Can chemotherapy alone eliminate the transmission of soil transmitted helminths? Parasit Vectors 2014; 7: 266.

20. Jia TW, Melville S, Utzinger J, et al. Soil-transmitted helminth reinfection after drug treatment: A systematic review and metaanalysis. PLoS Negl Trop Dis; 6. Epub ahead of print 2012. DOI: 10.1371/journal.pntd.0001621.

21. Vegvari C, Truscott JE, Kura K, et al. Human population movement can impede the elimination of soil-transmitted helminth transmission in regions with heterogeneity in mass drug administration coverage and transmission potential between villages: A metapopulation analysis. Parasites and Vectors 2019; 12: 1-12.

22. Saelens G, Gabriël S. Currently available monitoring and surveillance systems for taenia spp., echinococcus spp., schistosoma spp., and soil-transmitted helminths at the control/elimination stage: A systematic review. Pathogens; 9. Epub ahead of print 2020. DOI: 10.3390/pathogens 9010047

23. Tchuem Tchuenté LA. Control of soil-transmitted helminths in subSaharan Africa: Diagnosis, drug efficacy concerns and challenges. Acta Trop 2011; 120: 4-11.

24. Anderson RM, Turner HC, Truscott JE, et al. Should the goal for the treatment of soil transmitted helminth (STH) infections be changed from morbidity control in children to community-wide transmission elimination? PLoS Negl Trop Dis 2015; 9: 1-8.

25. Means AR, Ásbjörnsdóttir K, Mwandawiro C, et al. Sustaining Progress towards NTD Elimination: An Opportunity to Leverage Lymphatic Filariasis Elimination Programs to Interrupt Transmission of Soil-Transmitted Helminths. PLoS Negl Trop Dis 2016; 10: 1-4.

26. Anderson RM, Truscott JE, Pullan RL, et al. How Effective Is School-Based Deworming for the Community-Wide Control of SoilTransmitted Helminths? PLoS Negl Trop Dis; 7. Epub ahead of print 2013. DOI: 10.1371/journal.pntd.0002027.

27. Legge $H$, Kepha S, Prochazka M, et al. Implementer and recipient perspectives of community-wide mass drug administration for soiltransmitted helminths in Kwale county, Kenya. PLoS Negl Trop Dis 2020; 14: 1-18.

28. Mohammed KA, Deb RM, Stanton MC, et al. Soil transmitted helminths and scabies in Zanzibar, Tanzania following mass drug administration for lymphatic filariasis - A rapid assessment methodology to assess impact. Parasites and Vectors 2012; 5: 1-8. 